ISSN: $1130-3743$

\title{
EL SISTEMA EDUCATIVO COMO YACIMIENTO DE EMPLEO
}

\section{The educational system as source for employment}

\section{Le système éducatif comme gisement d'emploi}

\author{
Antonio J. COLOM CAÑELLAS y Catalina M. ${ }^{\text {a }}$ VILANOVA RIPOLL \\ Universidad de las Islas Baleares. Facultad de Educación. Campus Universitario. \\ Palma de Mallorca.
}

Fecha de aceptación definitiva: marzo de 2003

BIBLID [(1130-3743) 14, 2002, 121-150]

RESUMEN

Tras realizar una revisión al concepto de "Nuevo yacimiento de empleo" (origen, desarrollo, definición, funcionalidad, etc.), se plantean sus posibilidades en el campo educativo, dando lugar a dos perspectivas: la formación necesaria para desarrollar yacimientos de empleo y, considerar a la educación, al sistema educativo, como un nuevo yacimiento de empleo. Tomando esta segunda acepción, se realiza un estudio empírico de las posibilidades que oferta el sistema educativo de las Islas Baleares como nuevo yacimiento de empleo. En él encontramos posibilidades de empleo en tareas de servicios y en las actividades complementarias escolares, llegando a la conclusión de que el sistema educativo de las Islas Baleares (que sólo afecta a unos 850.000 habitantes), encierra una capacidad económica muy significativa (sobre los 20 millones de euros) cuyo capital podría desarrollarse socialmente si se cumplimentasen una serie de exigencias que analizamos en nuestro trabajo.

Palabras clave: nuevos yacimientos de empleo, educación y nuevos yacimientos de empleo, economía de la educación, actividades escolares complementarias, sistema educativo y educación no formal. 


\section{SUMMARY}

After revising the concept "New source for employment" (origin, development, definition functionality, etc.), its possibilities in the field of education are suggested, giving way to two perspectives: the training needed to develop sources for employment and, to consider education, the education system itself, as a new source for employment. Taking into consideration the latter proposal, we have carried out an empirical study of the possibilities offered by the system of education in the Balearic Islands, as a new source for employment. In it we can find possibilities for employment in the service sector and in the complementary activities of schools, thereby reaching the conclusion that the educational system of the Balearic Islands (which only affects around 850.000 inhabitants), entails a very significant economy capacity (of about 20 millions euros), which could be developed socially if a series of requirements that we are analysing in our work are met.

Key words: new sources for employment, education and new sources for employment, economy of education, complementaries activities schools, educational system and no formal education.

\section{SOMMAIRE}

Après la réalisation de la revisión du concept de "nouvelle gisement d'emploi" (origine, développement, definition, fonctionnalité...) etablisson leurs posibilites dans l'éducation, decuvrissant alors deux perspectives: la necessaire formation pour le développement de gisements d'emplois, et considerer à le sistème éducatif, a l'education, comme un nouvelle gisement d'emploi. Prètant attention a cet deuxième acception, on réalise un étude empirique sur les posibilites que à les Îles Baléares offrit le sustème éducatif comme nouvelle gisement d'emploi. Nous recontrons posibilites d'emploi dans travails subsidiaires et dans les activités ecoliers complementaires, de manière que le système éducatif a Baleàres (que seulement affecte a 850.000 habitants) apporte une capacité économique bien significative (sur à peu prés 20 milions euros); ce capital pour son developpement social requérit de diverses exigences qui nous analysons dans notre travail.

Mots clef: nouvelles gisements d'emplois, éducation et nouvelles gisements d'emplois, economie de l'éducation, activites écoliers complémentaires, système éducatif et éducation no formel.

En este artículo vamos a analizar el concepto de "nuevos yacimientos de empleo" a nivel general para luego incluirlo en el contexto educativo, lo que creemos representa una novedad en tal sector bibliográfico, máxime cuando aquí entenderemos la educación como verdadero yacimiento de empleo. Nuestra toma de postura se verá ampliamente informada en la realidad al realizar un estudio empírico sobre las posibilidades que el sistema educativo de las Baleares posee en el campo de la generación de nuevos empleos, diferentes, obviamente, a los propios 
del profesorado oficial. Con ello, por otra parte, introducimos un modelo de análisis y estudio que creemos puede ser aplicado a otras comunidades y a sus respectivos sistemas educativos. Las consecuencias que extraemos creemos pueden modificar aspectos teóricos interesantes referidos, fundamentalmente, a la educación no formal y de forma más concreta a la teoría económica de la educación.

\section{El CONTEXTO SOCIAL Y POLÍ́TICO DE LOS NUEVOS YACIMIENTOS DE EMPleO}

Se denominan "nuevos yacimientos de empleo" (NYE) a una alternativa de generación de empleo cuyos orígenes se encuentran en el Consejo Europeo celebrado en Copenhague en junio de 1992, y más aun, en una resolución del mismo por la que se solicitaba la realización de un Libro Blanco sobre estrategias para el desarrollo de la competitividad y el empleo. Este encargo dio lugar al texto realizado por una comisión que presidió Jacques Delors (1993), a la sazón presidente de la Unión Europea.

En el Informe Delors se nos evidencia cómo en los años ochenta y noventa del siglo xx se rompió el paralelismo entre crecimiento económico y generación de empleo, que era, como se sabe, típico de la era industrial; en este sentido no hay duda que la generación de las nuevas tecnologías en los procesos y modos de producción han originado nuevas perspectivas económicas que a su vez requieren de nuevas soluciones. Así, se nos dice que si bien entre 1972 y 1992 el PIB de los Estados Unidos aumentó un 70\%, el de Europa un 81\% y el de España un 103\%, paralelamente, el ritmo de creación de empleo para esta misma horquilla temporal fue, respectivamente, del 49\%, del 9\% y - para el caso español- de una pérdida de empleo del 0,3\%.

En los países integrados en la OCDE la tasa de ocupación -empleo en relación a la población potencialmente activa entre 15 y 64 años- era, en 1993, de sólo un 59\%, mientras que, en los Estados Unidos, alcanzaba el 72\%, y en el Japón, el 74\%. Si nos acogemos a la tasa de ocupación en el sector servicios - que es el contexto natural de aplicación de los nuevos yacimientos de empleo- J. Delors nos habla de un 33\% para el caso de los Estados Unidos, de un 30\% para el Japón y de un 26\% para Europa, (en el caso concreto de España no se alcanzaría el 20\%). Por tanto, si tenemos en cuenta las altas tasas de crecimiento en la Unión Europea, muy posiblemente podamos afirmar que el déficit de empleo en Europa no es fruto de su menor crecimiento sino de su menor capacidad de generar empleo, y sobre todo, de no saber generar empleo en el sector servicios, que es, por otra parte, el sector clave en la economía americana y japonesa (Cachón, 1998a; Montilla, 1996). Esta situación nos llevaría a considerar la crisis del trabajo desde un posicionamiento absolutamente revolucionario, tal como el logro de crecimiento sin empleo, lo que obviamente daría lugar a una nueva era histórica en la que el hombre, por fin, estaría liberado del trabajo por la mera razón de que a su vez el capital estaría librado de la carga de los trabajadores (Rifkin, 1996). 
No hay duda que las tesis de Rifkin se están haciendo patentes entre nosotros si bien no se ha llegado a los extremos enunciados. De lo que no hay duda es que nos encontramos ante una nueva tesitura que requiere de nuevas soluciones y de planteamientos más creativos. De todas formas las soluciones son difíciles y complejas pues en estas cuestiones no existen fórmulas milagrosas. La solución, de eso no hay duda, pasa, por dar una respuesta unificada de todos los países miembros de la Unión Europea sin caer en mecanismos estereotipados propios asimismo de la economía clásica (proteccionismo, aumento de los presupuestos estatales, aumento monetario...), que en poco tiempo arrastraría toda una serie de problemas de dificultosa solución, tales como la inflación, los desequilibrios externos y la grave crisis posterior que conllevaría aun a mayores cotas de desempleo. En cambio, una de las formas de paliar el desempleo se encuentra en las soluciones políticas y sociales. Por ejemplo, el Informe considera que Europa se encuentra en una buena posición para asumir políticas de empleo ya que dispone fundamentalmente de:

- Capital inmaterial, o lo que se ha venido en denominar capital humano; es decir amplios sectores de población con buena cualificación, con altos niveles educativos, con aptitud evidenciada para la innovación e integrados en un contexto cultural de larga tradición.

- Capital financiero y de una red bancaria ciertamente eficaz.

- Alta solidez de su modelo social, así como una adecuada concertación social, por lo que bien puede decirse que dispone de activos que sólo hace falta que fructifiquen en políticas de empleo eficaces.

A pesar de ser un espacio común, no debemos olvidar que en Europa coexisten diversas políticas de empleo - el mencionado Informe sería una referencia para iniciar su unificación- en relación con la tradición que aportan los diversos sistemas estatales de empleo; por tanto, se evidencia la necesidad de acciones coordinadas entre todos los Estados, a fin de lograr, en un futuro más o menos mediato, logros unificadores en cuanto a las políticas laborales. De principio se ve posible iniciar esta tarea si se incide de forma generalizada y coordinada sobre:

- La educación y la formación a lo largo de toda la vida.

- El aumento de la flexibilidad del mercado de trabajo.

- La confianza en la descentralización y en las iniciativas.

- La reducción de los costes del trabajo poco cualificado.

- La renovación profunda de las políticas de empleo.

- Ir al encuentro de las nuevas necesidades.

Es evidente que la mayoría de estas acciones dependen de las altas políticas estatales y de la propia política de la Unión Europea, sin embargo, la búsqueda de nuevas necesidades, al relacionarse con las iniciativas, máxime en un sistema descentralizado, son acciones que pueden caer dentro de la responsabilidad de los propios sujetos, es decir, de los trabajadores. Como afirma L. Cachón (1998a; 
1998b), nos encontramos en nuestra actual sociedad con muchas necesidades insatisfechas que son fruto de las nuevas variables que se están dando en nuestra actual sociedad. Así por ejemplo:

- La evolución de las formas de vida.

- Los cambios en la estructura y en las relaciones familiares.

- El aumento de la actividad profesional de las mujeres.

- Las nuevas aspiraciones y posibilidades de realización de la población anciana - como el actual fenómeno de la universidad para mayores (Colom y Orte, 2001).

- La acuciante necesidad de reparar y favorecer el estado de la naturaleza.

- La rehabilitación de barrios urbanos tradicionalmente desfavorecidos.

son sin duda ejemplos valiosos que nos evidencian el descubrimiento de necesidades perentorias de nuestra sociedad no plenamente satisfechas y que afectan, o pueden afectar, positivamente, al mercado laboral. Ahora bien, también cabe decir que estas nuevas perspectivas tropiezan con serios obstáculos tanto por lo que hace a la parte demandante como en el campo de la oferta. Es obvio entonces que la solución estribaría en la coordinación de la oferta y la demanda creando un referente o continuum de posibilidades entre la oferta íntegramente protegida por las subvenciones públicas, y la demanda, plenamente competitiva, ya que, de esta forma, surgiría una nueva economía de carácter social. Es decir, que la educación, la formación continuada, el aumento de la flexibilidad externa e interna, la descentralización, las iniciativas, la reducción de costes del trabajo poco cualificado, la renovación en profundidad de las políticas de empleo, etc., presuponen nuevas alternativas y estímulos a la creación de puestos de trabajo, sin depender del crecimiento (Cachón, 1998).

En tal contexto, y como nos dice el Libro Blanco, acaso una de las nuevas políticas de empleo a implementar para así roturar las tendencias de paro ${ }^{1}$ consista en "ir al encuentro de las nuevas necesidades ligadas a los cambios que acontecen en nuestras sociedades". Pues bien, los denominados Nuevos Yacimientos de Empleo, son una muestra de este nuevo rumbo que deben tomar las políticas laborales en el seno de la Unión Europea (Muiños y Cortegiano, 1998, 4).

1. Según el sindicato CC.OO. (citado por CACHÓN, 1999b, 18) se podrían generar en los próximos años hasta 100.000 puestos de trabajo. Incluso, el mismo autor, aumentando aun más las cifras, nos informa que en España, en estos momentos, de manera informal, más de 1.700.000 personas atienden a personas dependientes, de las cuales más de 700.000 dedican a tales menesteres un mínimo de 40 h a la semana. 


\section{LOS NUEVOS YACIMIENTOS DE EMPLEO: UNA APROXIMACIÓN FUNCIONAL}

De principio, y tal como se desprende de nuestras últimas palabras, los nuevos yacimientos de empleo se centrarían en cubrir necesidades de trabajo no satisfechas en nuestra sociedad. De hecho, se confirma la hipótesis de que estas "nuevas necesidades" surgen fundamentalmente en el sector de los servicios, a fin de dar respuesta a necesidades que se manifiestan tanto en el plano de los individuos como colectivamente. Acaso, junto a ellos, la insosteniblidad del desarrollo sea un nuevo vector — una nueva necesidad - que también se considera acuciante hoy en día y que requiere de nueva mano de obra para su control o corrección.

Ahora bien, las necesidades actuales son fruto de cambios radicales que se están dando en la actual sociedad. Las causas son múltiples y las nuevas tecnologías de la información juegan también su papel ya que, según J. J. Duque Murua (1999, 293), el contexto natural de los nuevos yacimientos de empleo es la sociedad de la información que, como se sabe, ha dado lugar a la sociedad del conocimiento, en donde la gestión del ocio o la gestión cultural -la gestión, en definitiva, de la calidad de vida- se presenta como una de las necesidades más perentorias. No obstante, el Informe Delors, nos amplía el horizonte causante de los nuevos yacimientos de empleo al hablar en concreto de los siguientes factores, a saber:

- Demográficos (envejecimiento de la población).

- Sociales (con la incorporación de la mujer al mundo laboral).

- Profundas transformaciones familiares (familias mono parentales).

- Incremento de la urbanización de la población (dando lugar a múltiples problemas de viviendas y de planificación urbanística en las ciudades).

- Nuevas necesidades de carácter cultural (centradas en gran parte en formas nuevas de ocio).

G. Esping Anderson (1993) afirma que en Europa, tradicionalmente, los servicios se han considerado, o bien, socio-públicos (sanidad, educación, bienestar social), donados por el Estado, o bien, privados o personales — orientados por lo general a formas clásicas de ocio, a la restauración, etc. Es decir, Europa no ha sabido anticiparse a los cambios convulsivos que han ilustrado la vida urbana, social y familiar. En cambio, los nuevos yacimientos de empleo se centran en el potencial laboral que manifiestan los cambios de la sociedad actual. Así, el envejecimiento de la población, puede generar la necesidad de empleo en cuanto atención específica para este sector; lo mismo podríamos decir de la incorporación de la mujer al trabajo, que obliga a encontrar sustituciones personales para las labores domésticas, o para la atención de sus hijos. Incluso las limitaciones de ayudas públicas del Estado del Bienestar, que se resiente de sus capacidades económicas, pueden ser las causantes de nuevas demandas de servicios que deberán ser ofertados privadamente. Por otra parte, el crecimiento de la población en las ciudades posibilita también necesidades de vivienda y de servicios urbanos al mismo tiempo 
que genera focos de marginación que deben ser controlados y tratados convenientemente para el logro de su futura reinserción, etc.

Como se ve, las nuevas perspectivas sociales crean nuevas necesidades laborales, siendo en consecuencia estos nuevos locus el nicho natural de los denominados nuevos yacimientos de empleo ${ }^{2}$. Ahora bien, para que los nuevos yacimientos de empleo se transformen en realidades empresariales y/o laborales deben manifestar estas características (Jiménez, Barreiro y Sánchez, 1998, cap. II): estar destinados a satisfacer nuevas necesidades sociales, estar configurados como mercados incompletos, estar territorialmente definidos y ser, por último, intensivos en el empleo. Lo que implica dificultades - definir una necesidad real-o definir los "mercados incompletos", que serían aquellas áreas de empleo en las que el mercado instituido renunciase a intervenir al no existir una demanda solvente y declarada, lo que hace que estas bolsas laborales muchas veces se confundan con las prestaciones sociales, propias del Estado del bienestar. En consecuencia, hablar de yacimientos nuevos de empleo implica no sólo detectar o descubrir necesidades sino también ir removiendo los obstáculos que impiden la solvencia de la demanda, (Jiménez, Barreiro y Sánchez, 1998, cap. II).

En consecuencia, L. Cachón (1999b, 86) nos anuncia que los nuevos yacimientos de empleo son el resultado de "ir al encuentro de las nuevas necesidades laborales ligadas a los cambios que acontecen en nuestras sociedades». En el mismo sentido, si bien de forma más amplia, M. ํ J. Bono y E. Jiménez Hernández (1997, 1), nos afirman que los NYE son "las nuevas necesidades sociales originadas en las profundas transformaciones demográficas, sociales y culturales de las últimas décadas, cuya satisfacción no es bien resuelta ni por el sector público ni por las ofertas mercantiles y que se expresa en una gran demanda insatisfecha de servicios". Por su parte, E. Jiménez, F. Barreiro y J. E. Sánchez $(1998,18)$ nos dicen que "son aquellas actividades destinadas a satisfacer nuevas necesidades sociales que actualmente se configuran como mercados incompletos, sean intensivos en empleo y tengan un ámbito de producción/prestación definido en el territorio". Definición que asimismo hace suya R. Rubio (2000).

En su formato original, es decir en el Informe Delors, los nuevos yacimientos de empleo se definían diciendo que son «ámbitos de actividad económica que

2. En un nuevo documento (COMISÍ́n EuropeA, 1995, 28), y en referencia a los nuevos yacimientos, se afirma que "paulatinamente deberán sustituir a una parte de las prestaciones sociales" de tal manera que a la sombra de los NYE se intuye una concepción del servicio público que sin dejar de ser servicio público se verá desarrollado desde el sector privado. Es decir, los servicios públicos o producto de los impuestos ciudadanos se irán poniendo de cada vez más en manos de las empresas privadas que se reconvertirán en prestadoras directas de los servicios a los usuarios. O sea, que se crea ocupación convirtiendo en capital — poner en el mercado- el conjunto de recursos públicos con determinada función asistencial, lo que implica descubrir nuevos yacimientos de empleo en la medida en que también se ponen al descubierto los capitales públicos. Lo que a su vez conlleva un planteamiento adicional, y es que, si se trata de privatizar de alguna forma servicios públicos, indudablemente la medida asistencial debe moverse y desarrollarse en el plano local. 
ANTONIO J. COLOM CAÑELLAS Y CATALINA M. ${ }^{a}$ VILANOVA RIPOLL EL SISTEMA EDUCATIVO COMO YACIMIENTO DE EMPLEO

vienen a satisfacer estas necesidades nuevas de nuestras sociedades, explotando de manera activa y positiva el filón económico y de empleo que se esconde en esos cambios sociales, aprovechando las oportunidades de crecimiento y de empleo que pueden venir a satisfacer las necesidades que surgen de esas transformaciones sociales". O como afirma M. Domínguez $(2001,18)$, con los nuevos yacimientos de empleo se trata de aprender a emprender. Creemos que aquí está el quid de la cuestión ya que los NYE no sólo implican ocupar un espacio laboral sino que, previamente, se trata de emprender la búsqueda de su hallazgo.

Búsqueda que en el Informe Delors se resuelve con el encuentro de cuatro tipos de nuevos yacimientos de empleo que responden a otras tantas necesidades, a saber: los servicios de proximidad o llamados también "servicios de vida diaria", las mejoras de las condiciones de vida, los servicios culturales y de ocio, y los servicios ambientales, que a su vez darían lugar a los siguientes ámbitos de empleo:

\begin{tabular}{|ll|}
\hline SERVICIOS DE PROXIMIDAD & MEJORA DE LAS CONDICIONES \\
& DE VIDA \\
Servicios a domicilio & Mejora de vivienda \\
$\begin{array}{l}\text { Cuidados de niños } \\
\text { Nuevas tecnologías de la información }\end{array}$ & $\begin{array}{l}\text { Seguridad } \\
\text { Transportes colectivos locales } \\
\text { y de la comunicación }\end{array}$ \\
$\begin{array}{l}\text { Ayudas a jóvenes con dificultades } \\
\text { Inserción de jóvenes, etc. }\end{array}$ & $\begin{array}{l}\text { públicos urbanos de espacios } \\
\text { Comercios de proximidad }\end{array}$ \\
SERVICIOS CuLTURALES & SERVICIOS DE MEDIO AMBIENTE \\
Y DE OCIO & Gestión del agua \\
Gestión de residuos & Control de la contaminación \\
Turismo & y de las instalaciones \\
Sector audiovisual & correspondientes \\
Valorización del patrimonio & Protección y mantenimiento \\
cultural & de zonas naturales \\
Desarrollo de las culturas & \\
locales & \\
\hline
\end{tabular}

Así pues, el Libro Blanco, cita 17 nuevos yacimientos de empleo, que como se ve, no obedecen a criterios sistemáticos o rígidos pues entre ellos encontramos algunos de tipo sectorial - el turismo sería una clara expresión de lo afirmadootros son horizontales o transversales - es decir, válidos para cualquier sector económico- como puedan ser las nuevas tecnologías; otros suponen transformar viejas actividades propias del sector secundario, como pueda ser la modificación de viviendas y la generación y actualización de barrios; otros pueden ser considerados como resultado de las transformaciones del Estado del bienestar tal como sería el caso de los servicios a domicilio, encontrando, por fin, otros nuevos yacimientos que estarían en relación a nuevos problemas sociales como puedan ser los propios de la inserción de jóvenes en el mundo laboral, o el cuidado y atención a la 
tercera edad, que, como se intuye, es también fruto de los cambios sociales habidos en estos últimos años.

Cabe decir que a los 17 yacimientos de empleo que reseñara el informe propiciado por J. Delors, la Unión Europea ha añadido dos más (Cachón, 1999b, 86), a saber: las energias renovables y el deporte. De todas formas parece ser que no serán los últimos, pues se ve la necesidad de ir ampliando los espacios de empleo. Es el caso, por ejemplo de L. Cachón (1999a, 126), que concreta la agricultura ecológica, la industria agroalimentaria de carácter artesanal, la reforestación y las ONGs como otros yacimientos a tener en cuenta, o de M. Domínguez (2001, 20) que insiste en el animador teatral para jóvenes como otro posible yacimiento de empleo. Nosotros mismos, más adelante, propondremos un nuevo yacimiento de empleo centrado en los nuevos puestos laborales que se desprenden del sistema educativo formal.

Creemos que con las definiciones expuestas y los ejemplos aportados se tienen ya todas las variables que nos dan pie para entender conceptualmente lo que es un nuevo yacimiento de empleo. Sin embargo, queremos insistir en que la cuestión de los NYE es algo más profundo que una búsqueda - fundamentalmente en el área de los servicios- de nuevas posibilidades de trabajo en el sentido de que obedecen a un cambio profundo y aun a una revolución a la hora de entender la economía actual. Téngase en cuenta que desde las posiciones neoliberales la cuestión del empleo defiende un crecimiento económico de las leyes del mercado, olvidándose de los factores sociales, lo que por lo general conlleva una gran fractura social. En cambio, los nuevos yacimientos de empleo se incardinan en otro planteamiento de carácter abiertamente social, ya que como dice Rifkin (1996), las transformaciones que a la larga supondrán las nuevas tecnologías informáticas hará que las nuevas formas de trabajo se irán gestionando cada vez más desde la sociedad civil, ya que las relaciones humanas nunca se verán sustituidas por ningún tipo de artefactos. Éste es el cambio que debe valorarse a la hora de hablar de nuevos yacimientos de empleo, es decir, las leyes del mercado a la hora de generar empleo se ven sustituidas por unas demandas sociales que son realmente las que generan los nuevos puestos de trabajo.

Es evidente entonces que con la aparición y generalización, cada vez más amplia, de los nuevos yacimientos de empleo se tengan que ir modificando las nuevas políticas de fomento de empleo que ahora tendrán que contemplar toda la fenomenología que conllevan las nuevas transformaciones sociales en el mundo laboral.

¿Estamos ante una actitud voluntarista? ¿Pueden los nuevos yacimientos de empleo ser una panacea para las situaciones de paro laboral? Los nuevos yacimientos no son fruto del voluntarismo aunque ciertamente se necesita voluntad de descubrir, de emprender nuevas perspectivas laborales, esto está fuera de toda duda, y efectivamente, los NYE, no son la solución única al problema del paro. Sin embargo, sí que ayudan a paliarlo, e indudablemente, es una apuesta de futuro que puede dar pie a mucha gente para iniciarse, bien por primera vez, o de nuevo, en 
el mundo laboral. Las cifras no son optimistas del todo pero algo sí que se está mejorando. En este sentido, P. Gomis $(2000,116)$ afirma que en el seno de la Unión Europea y debido en gran medida a los nuevos yacimientos de empleo el paro disminuyó en 1.800 .000 personas lo que supone un aumento laboral del 1,2\%. Asimismo, en mayo de 1999, el desempleo en el seno de la Unión alcanzaba el 9,4\%, frente al 10,1\% que se había logrado en el mismo mes de 1998. En estos momentos, cuatro años más tarde la tasa de paro ha descendido hasta el 8\%.

\section{LA EDUCACIÓN Y LOS NLEVOS YaCiMIENTOS DE EMPLEO}

Los nuevos yacimientos de empleo se relacionan con la educación a través de la necesidad de formación que muchas veces se requiere para desarrollarlos laboralmente. Para nosotros ésta sería la perspectiva clásica de la cuestión porque se puede dar otro sentido en las relaciones educación-nuevos yacimientos de empleo, que sería entender la educación como objetivo laboral, es decir, entender la educación como un nuevo yacimiento de empleo. Éste es el motivo del presente artículo ya que no hemos visto bibliográficamente, ni tan siquiera en el plano internacional, reflejada la comprensión de la educación como yacimiento de empleo.

Como se sabe, debido fundamentalmente a la aparición de la educación no formal y por tanto al descubrimiento de las múltiples vertientes y posibilidades de los programas educativos fuera de la escuela, los centros escolares se han visto en la necesidad de ampliar sus horarios y convertirse ellos mismos en promotores de ofertas educativas (y otros servicios) para sus propios alumnos. La competencia entre escuela pública y privada, el desarrollo de la sociedad civil y la participación de los padres en los asuntos escolares y en la gestión educativa a través de las AMPA (asociaciones de madres y padres de alumnos), han sido otras de las variables que han hecho que la escuela sea un foco de oferta de programas y servicios complementarios.

De esta forma, los centros educativos se conforman como instituciones que ofertan nuevas formas de empleo, en base a cumplimentar dos objetivos:

- Aumentar la oferta educativa y formativa de carácter complementario (en el ámbito deportivo, cultural, artístico, etc.).

- Ofrecer a las familias un tipo de servicios para así facilitar la organización doméstica de las mismas (servicios de comedor, de transporte escolar, etc.).

De ahí, entonces, que a partir de esta doble funcionalidad contractual de las escuelas o de las AMPA - oferta escolar complementaria y servicios de apoyopodamos considerar a los sistemas educativos como un nuevo yacimiento de empleo, cuya oferta en cuanto a puestos de trabajo puede ser realmente importante. Sin embargo, y como decíamos, no hemos visto tal consideración en la bibliografía, es decir, hasta el momento no se ha concebido que el sistema educativo pueda ser considerado como un yacimiento de empleo de potencialidad manifiesta. 
Pues bien, llegados a este punto, debemos decir que se pretenderá estudiar el ámbito escolar desde tales perspectivas para coadyuvar así a la posibilidad de generar un nuevo filón de empleo. Así pues y a continuación, para poder comprobar tal aserto, intentaremos estudiar esta realidad en las escuelas públicas y privadas. No hay duda que los resultados de nuestra búsqueda serán aportaciones para el logro de una teoría económica de la educación más completa.

4. Estudio acerCa de los NueVos yacimientos DE EMPLEO EN El SISTEMA EDUCATIVO DE LAS BALEARES

A partir de aquí daremos cuenta de una investigación encaminada a detectar posibilidades de nuevos empleos en el sistema educativo de las Islas Baleares, o diciéndolo de otra forma, intentaremos evidenciar que existe realmente la opción de considerar el sistema educativo como un nuevo yacimiento de empleo.

\subsection{Objetivos e hipótesis}

El objetivo de esta investigación es ver si se puede considerar al sistema educativo como un nuevo yacimiento de empleo. Es decir, se trata de descubrir la capacidad que posee el sistema educativo de ofertar puestos de trabajo, independientemente del de los docentes que marca la ley.

Fruto de tal objetivo será la siguiente hipótesis que enmarcará el proceso investigacional; la formulamos afirmando que si, que efectivamente, el sistema educativo genera puestos de trabajo que no son propios de los docentes que imparten los cursos y las asignaturas obligatorias y/u optativas de acuerdo con la legislación vigente. O sea, si se quiere plantear de otra manera: consideramos al sistema educativo como un nuevo yacimiento de empleo, por lo que se puede integrar en el contexto teórico que nos ha servido hasta ahora de enmarque. De tal hipótesis general extraeremos las siguientes subhipótesis:

- Los colegios privados y concertados generan mayor número de nuevos puestos de trabajo. La razón de tal formulación estriba en la creencia de que por la competencia que se da entre ellos, y en referencia a la enseñanza pública, ofertan más actividades extraescolares que los colegios públicos. Además, un control no tan estricto por parte de la Administración, puede, asimismo, facilitar la oferta de servicios y actividades de formación.

- Los colegios instalados en la capital, Palma de Mallorca, ofrecen, significativamente, mayor número de actividades y servicios extraescolares que los demás. La razón de tal formulación estriba en la consideración de que el clima urbano requiere y contempla más perentoriamente la necesidad de formación adicional; además, la mayor concentración de colegios y de población posibilita, por una parte, mayores cotas de competencia y mayor facilidad de contratación de profesionales. 
- Los centros públicos primarios ofrecen mayor número de servicios y de actividades complementarias que los de secundaria. La razones que aducimos se centran en el hecho de considerar que en la enseñanza primaria, la edad de los niños y su menor autonomía, exige, acaso, de una estancia mayor en el centro en función de los horarios de los padres (para que no estén solos en casa, para que no hagan el trayecto escuela-hogar solos, etc.); además, al exigir la secundaria más horas de dedicación, hace que, en estas edades, se vayan abandonando las actividades extraescolares para concentrarse más en los estudios.

- No consideramos que haya diferencias entre la primaria y la secundaria en los colegios privados y concertados a la hora de ofertar actividades y servicios complementarios a sus alumnos. Lo fundamentamos en el hecho de que tanto en un nivel como en otro siguen presentándose los mismos argumentos competenciales en referencia a la enseñanza publica, junto con las otras razones hasta aquí aducidas.

Cabe señalar que en la investigación utilizaremos dos constructos, a saber:

a) Servicios, o aportes escolares a través de profesionales que no tienen incidencia alguna sobre la formación; nos referiremos a chóferes, monitores de autocar, cocineros, ayudantes y pinches de cocina, monitores de comedor, personal de limpieza y de mantenimiento, etc.

b) Actividades complementarias, o aportes escolares, a través de profesionales cuya actividad es complementaria a la formación que reciben los niños en el centro escolar; nos referimos, por ejemplo, a profesores, o monitores deportivos, de informática, de teatro... o cualquier otra actividad formativa no reglada por la legislación y que no imparten los profesores titulares de los centros.

\subsection{Proceso seguido}

El proceso investigacional ha consistido en desarrollar los siguientes pasos, en el período comprendido entre septiembre del 2001 y principios de marzo del año 2002:

- Dividir las Baleares en cuatro territorios, a saber: Palma de Mallorca, resto de Mallorca, Menorca e Ibiza y Formentera.

- Conocer la población real y número de centros de las Baleares en función de los territorios reseñados. Esta información nos fue dada por la Conselleria de Cultura, Educació i Esports, del Govern Balear.

- Sobre tales parámetros definir la muestra objeto de la investigación.

- Realizar un cuestionario a través de diversos ítems cerrados y abiertos para solicitar información sobre los servicios y actividades complementarias que ofertaban tales centros. 
- Entrenar a los colaboradores —estudiantes de Pedagogía - en los objetivos, hipótesis, modos de contactar con los centros educativos y de someter a sus responsables a la entrevista que exige el cuestionario.

- Visitar los centros de la muestra y entrevistarse con sus responsables a fin de que contestasen al mencionado cuestionario.

- Unos treinta centros - un nuevo muestreo al azar- fueron controlados a través de las informaciones que la Conselleria pertinente tenía sobre las actividades que desarrollaban, no dándose, en este sentido, ninguna diferenciación con la información que fuimos recabando.

- Conjuntar los resultados por zonas geográficas y por tipos de centros: públicos y privados-concertados.

- Extraer resultados estadísticos en función de los resultados obtenidos.

- Extraer conclusiones en referencia a los objetivos e hipótesis propuestas.

\subsection{Incidencias}

Cabe decir que los entrevistadores se presentaban a los centros sin decir la finalidad concreta del trabajo - en referencia a los nuevos yacimientos de empleo- y sin aclarar estrictamente quién era el organismo que patrocinaba la investigación. Los motivos que se daban para realizar la investigación eran pues de otra índole, fundamentalmente, el interés que socialmente despiertan, cada vez más, las actividades complementarias, lo que ya era suficiente para que los colegios tuviesen una buena e incluso muy buena disposición para contestar. Si algún centro solicitaba quién patrocinaba la investigación, siempre fue suficiente contestar que era de parte de la Conselleria, sin especificar cuál era realmente. Creemos que con tales actitudes se ganaba en objetividad y se contaminaban menos nuestros objetivos.

Los centros nos acogieron con amabilidad y comprensión, y con una aceptación total de las exigencias que de tiempo e información suponía la aplicación del cuestionario. Cabe decir que los centros se sorprendían por el tipo de cuestiones y preguntas que se les demandaba ya que por lo general nos decían que era la primera vez que alguien se preocupaba por estas cuestiones... No hubo pues ningún incidente negativo en la relación con los centros Por tanto, no cabe señalar ningún problema, ni de rechazo, ni de desconfianza, ni de negativas, etc. En todo caso, advertir que en los centros de secundaria públicos y en los privados-concertados la relación se mantuvo en un nivel más formal - fueron más parcos en las respuestas - pero siempre con absoluta y exquisita corrección.

En todo caso cabe decir que si la muestra de los colegios privados-concertados no se ha podido cumplimentar en su totalidad ha sido debido a que cuando entrábamos en contacto con tales centros no nos contestaban el mismo día dándonos hora para hacerlo en otras fechas, casi siempre muy alejadas (promedio de un mes); esto ha hecho que la muestra se resintiera sobre todo en aquellos territorios que requerían desplazamientos (resto de Mallorca y no hablemos ya de Menorca e 
Ibiza). Algo de esto también ha pasado en los institutos de secundaria, si bien nunca de forma tan acusada.

\subsection{Estudio poblacional}

La presente investigación se desarrolla en el seno del sistema educativo de las Islas Baleares, lo que supone los territorios de Mallorca, Menorca, Ibiza y Formentera. El 65,72 (255) de los centros de las Baleares son públicos y el 34,28\% (133) privados, mientras que 84.857 alumnos $(63,84 \%)$ asisten a centros de enseñanza pública, y el resto, o sea, 48.057 alumnos (36,16\%), a centros privados. Por tanto, grosso modo, podemos decir que la población del sistema educativo de las Baleares por lo que hace a alumnos y centros de propiedad pública y privada-concertada está en relación dos a uno (2/1) a favor de los centros (y alumnos) públicos.

\subsection{Selección de la muestra}

Por lo que se refiere a la muestra hemos intentado mantener la misma proporción que encontramos a nivel poblacional, eligiendo los centros al azar con la pretensión de entrevistar al 50\% de los mismos, lo que no se consiguió, debido a las dificultades, ya mencionadas, que encontramos en los centros privados. En definitiva hemos visitados:

a) Sistema público primario

- Colegios Públicos de Palma: 17 sobre un total de 30. Representa el $56,66 \%$ de los centros.

- Resto de Mallorca: 41, lo que representa, en cuanto a centros, el $34,16 \%$.

- Menorca: 8 sobre un total de 18, lo que supone el 44\%.

- Ibiza-Formentera: 15, lo que supone el 45,45\% de los centros.

b) Centros de secundaria públicos

- Palma: 8 centros de los 15 que existen en la ciudad, lo que supone un $53,33 \%$.

- Resto de Mallorca: 9, que supone el 37,5\% de los institutos.

- Menorca: 4 de los siete institutos públicos, lo que supone un 57,14\% de los centros.

- Ibiza-Formentera: 5, lo que supone el 75\% del total.

c) Sistema concertado-privado

- Palma: 12 centros, lo que supone un 24\% de los centros.

- Resto de Mallorca: 5, que suponen sólo un 7,57\%. de los centros.

- Islas menores: 4 centros, o sea, el 23,52\%

La muestra de escuelas del sistema público de educación, por lo que se refiere a la enseñanza primaria, es del 40,29\% de los centros y la de secundaria alcanza el 
$50 \%$ de los mismos. Por tanto la muestra analizada del sistema público de educación en función del detectaje y definición de los nuevos yacimientos de empleo, ha sido del 41,9\% de los centros (107 de 255). En cuanto a los centros privados-concertados hemos logrado el 15,78\% de los mismos. En total, la muestra lograda para todas las Baleares ha sido de 128 centros, lo que supone el $32,9 \%$ de los mismos.

Ahora bien, las actividades extraescolares así como los servicios que prestan los centros inciden sobre los alumnos por lo que acaso más importante sea saber el número de alumnos que han sido objeto de nuestro estudio, y en este sentido, cabe decir que los centros visitados se corresponden con 53.719 alumnos de los 132.912 posibles, o sea, un 40,41\% del total de las Baleares así distribuidos:

\begin{tabular}{lcc} 
Enseñanza pública & Primaria & Secundaria \\
\hline Palma & $62,70 \%$ & $47,02 \%$ \\
Resto de Mallorca & $41,75 \%$ & $45,13 \%$ \\
Menorca & $54,02 \%$ & $62,74 \%$ \\
Ibiza/Formentera & $52,70 \%$ & $51,53 \%$ \\
TOTAL & $48,88 \%$ & $48,58 \%$
\end{tabular}

Como se puede observar, las muestras para ambos grupos (de primaria y de secundaria) de Ibiza/Formentera y del resto de Mallorca están perfectamente compensadas, existiendo mayor diferenciación por lo que respecta a Menorca ( 8 puntos) y sobre todo Palma ( 15 puntos). No obstante, teniendo en cuenta que nos movemos alrededor del 50\% de la población y que, al final, ambas muestras tota les están absolutamente compensadas (sólo una diferencia de un 0,30\%), creemos que los resultados no ofrecen duda alguna en cuanto a su fiabilidad.

En cuanto a la enseñanza privada-concertada el muestreo, en referencia a los alumnos, ha sido el siguiente:

\begin{tabular}{lc} 
Enseñanza privada-concertada: & Primaria + Secundaria \\
\hline Palma & $44,32 \%$ \\
Resto de Mallorca & $11,93 \%$ \\
Menorca & $24,94 \%$ \\
Ibiza/Formentera & $25,67 \%$
\end{tabular}

Sólo en Palma hemos podido mantener el mismo parámetro de muestreo que en la enseñanza pública, bajando ostensiblemente en el resto de territorios, debido a las dificultades ya aducidas. No obstante se han podido estudiar los servicios y actividades que disfrutan más de una cuarta parte de esta población estudiantil. En total, y como ya habíamos mencionado, hemos analizado el 40,41 de la población escolar total de primaria y secundaria (53.719 alumnos), lo que creemos fiabiliza nuestros resultados, máxime cuando la elección de centros ha sido totalmente aleatoria. 


\section{CONCLUSIONES Y COMENTARIOS}

En primer lugar, vamos a responder lo más concretamente posible a las hipótesis y subhipótesis que nos han servido de guía y orientación a la hora de desarrollar nuestro trabajo, para luego, en una segunda fase, ampliar y comentar más ampliamente los resultados hallados.

En referencia a la única hipótesis, y con los resultados obtenidos, debemos contestar afirmativamente a su formulación, es decir, que efectivamente, el sistema educativo genera otros puestos de trabajo diferentes a los cubiertos por la plantilla oficial docente que imparte los cursos y asignaturas obligatorias y/u optativas de acuerdo con la legislación vigente. Puede pues considerarse un nuevo yacimiento de empleo, e integrarse entonces en el contexto teórico que nos ha servido de enmarque. Ahora bien, esta afirmación requerirá de algún comentario así como de algunas precisiones que sin duda nos hará costreñir nuestra anterior afirmación. Volveremos sobre ello más adelante.

- Por lo que respecta a la primera subhipótesis - los colegios privados y concertados ofertan mayores posibilidades a la hora de generar nuevos puestos de trabajo- cabe contestar afirmativamente ya que hemos visto que los colegios privados y concertados ofertan mayor número de actividades complementarias que las escuelas públicas, si bien la primaria pública casi alcanza los mismos parámetros. Además, si tenemos en cuenta que, en gran medida, los servicios de las escuelas públicas están en manos de funcionarios (caso de los porteros, administrativos, del personal de limpieza...), no hay duda que los centros privados ofrecen un gran yacimiento de empleo, exactamente en esta área, es decir, en la de los servicios complementarios. Por tanto, en cualquier término se cumplimenta la subhipótesis. Es decir, que tanto a nivel de actividades complementarias como en el plano de los servicios, los centros concertados-privados ofertan más posibilidades de trabajo que los públicos.

- En referencia a la segunda subhipótesis - los colegios instalados en Palma de Mallorca ofertan significativamente mayor número de actividades y servicios extraescolares que los colegios y escuelas de las islas menores y del resto de la isla de Mallorca- cabe decir que no se ha visto confirmada. Sólo quedaría parcialmente aceptada para la enseñanza secundaria pública de Ibiza y la concertada-privada de Menorca en donde, en ambos casos, sî se denota una gran diferencia de actividades en favor de los centros homónimos de Palma.

- La tercera hipótesis - los centros de nivel primario públicos aportan más actividades complementarias que los de secundaria- se cumplimenta sin duda alguna siendo las diferencias abismales en todas las zonas geográficas. Obviamente, al ser por lo general los centros concertados-privados de carácter mixto - primaria y secundaria-, la subhipótesis no se puede demostrar en estos centros. 
- La cuarta subhipótesis - no hay diferencias entre la primaria y la secundaria en los colegios privados y concertados a la hora de ofertar actividades y servicios complementarios a sus alumnos, se refrenda totalmente ya que los servicios y actividades de estos centros se ofrecen por un igual y paralelamente a los dos niveles de enseñanza.

Un gráfico resumen de los promedios de las actividades que se realizan por centro en el plano de la complementariedad académica nos refrenda en lo afirmado:

\begin{tabular}{lccc} 
& \multicolumn{2}{c}{ Enseñanza Pública } & Enseñanza Concertada \\
\cline { 2 - 4 } Zona & Primaria & Secundaria & \\
\hline Palma & 7 & 3,87 & 8,25 \\
Resto Mallorca & $7 ’ 02$ & 2,89 & 7,4 \\
Menorca & 7,62 & 2,05 & 3,5 \\
Ibiza/Formentera & 6,20 & 0,6 & 8
\end{tabular}

Vemos cómo, efectivamente, la enseñanza concertada, oferta a simple vista mayores niveles de actividad que la enseñanza pública (que separa sus niveles de primaria y secundaria). Vemos también, que no necesariamente en Palma se oferten mayores cantidades de actividades extraescolares, y que, en todo caso, las diferencias no son significativas. Asimismo, también se denota, con estas cifras, la diferencia significativa a todas luces entre la enseñanza primaria y la secundaria pública en cuanto al número de actividades. En el caso en el que quisiéramos que la enseñanza pública apareciese conjuntada - primaria más secundaria- como en la enseñanza concertada, las cifras acentuarían aun más las diferencias a favor de la enseñanza concertada

Enseñanza pública primaria + secundaria. Índices de actividades

\begin{tabular}{lr}
\hline Palma & 6 \\
Resto de Mallorca & 6,28 \\
Menorca & $5^{\prime} 91$ \\
Ibiza/Formentera & 4,8
\end{tabular}

Es decir, sólo en el caso de Menorca, la enseñanza pública desarrollaría mayor número de actividades que la escuela concertada-privada. Como conclusión, y a nivel general, podemos decir que, efectivamente, la enseñanza concertada aporta, en proporción, mayor número de actividades extraescolares y, en consecuencia, mayor número de puestos de trabajo que la enseñanza pública. Decir, en todo caso, porque creemos que el dato es importante, que son las escuelas de primaria públicas, las únicas que, de alguna forma, pueden competir casi de igual a igual con el número de actividades que desarrollan las escuelas concertadas-privadas.

Así las cosas y como decíamos, podemos reiterar que, efectivamente, el sistema educativo se nos presenta como un yacimiento de empleo, al que, sin embargo, hemos de caracterizar bastante negativamente, ya que es: 
- Un yacimiento de empleo con muchas dificultades (como veremos) en un mercado de trabajo excesivamente complejo por los diferentes perfiles profesionales implicados, lo que, en consecuencia, supone dificultades para formalizar programas de formación.

- Además, y en general, muchos de los hipotéticos trabajos no ocupan las horas de una jornada laboral normal, por lo que dificulta la contratación de personas con dedicación exclusiva a tales tareas. Es evidente que con un circuito de colegios, los responsables de muchas actividades podrían a lo sumo completar media jornada laboral -las tardes- ya que por las mañanas difícilmente podrían trabajar en tales menesteres. En consecuencia destacaríamos, además, la dificultad de poseer plena dedicación en gran parte de los trabajos ofertados, por lo que los niveles de trabajo serían bastante precarios.

- Otra de las dificultades que vemos se refiere a las entidades contratantes (en general las asociaciones de padres, que como tales no tienen posibilidad de contratar trabajadores legalmente). Así pues, sería necesario cambiar este aspecto ya que, para los colegios públicos, según la actual legislación, sólo es la Conselleria correspondiente quien tendría tal potestad. En el caso de los colegios concertados privados, los propios colegios podrían contratar en función de las aportaciones de las AMPA, aunque, claro está, esto supondría declarar nuevas entradas económicas. Es decir, que en este yacimiento de empleo se dan y se están dando altos niveles de economía sumergida.

- Como tal yacimiento, el sistema educativo no es novedoso ya que surgió con anterioridad a la filosofía de los nuevos yacimientos de empleo, propia del Informe Delors. Es decir, sus puestos de trabajo, en muchas ocasiones, se han ido ocupando de forma voluntarista, con la filosofía de "hacer más", de "aportar algo más a los alumnos" y no tanto, desde una perspectiva profesional. Es decir, contamos con otro hándicap a tener en cuenta. Además, muchos de los monitores encuentran en este trabajo gratificación porque ello les supone no sólo desarrollar su afición sino también sentirse reconocidos, y por tanto, reconfortados psicológicamente, por lo que consideran que tales actividades más que un trabajo es una forma de desarrollo personal. En consecuencia, deberíamos añadir como características de este yacimiento de empleo la falta de tradición profesional.

- Por otra parte, los trabajos que podían cumplimentarse a través de toda la jornada laboral, suelen conseguirse mediante oposición, como es el caso de los porteros de los centros, o de los administrativos de los IES. Es decir, que este yacimiento de empleo sufre una alta competencia funcionarial por lo que, entre una cosa y otra, se trata de un yacimiento en gran parte ya ocupado.

Por tanto, una definición, o descripción ajustada, del sistema educativo como nuevo yacimiento de empleo debiera fundamentarse a partir de las notas 
con las que lo hemos ido caracterizando hasta el momento y que ahora presentamos sistemáticamente:

- Yacimiento de empleo con muchas dificultades.

- Con un mercado de trabajo excesivamente complejo por los diferentes perfiles profesionales implicados.

- Dificultad, en consecuencia, de formalizar, programas de formación.

- Dificultad de poseer plena dedicación en gran parte de los trabajos ofertados.

- Altos niveles de economía sumergida.

- Falta de tradición profesional.

- Actividades desarrolladas desde el voluntarismo o el bobby.

- Alta competencia funcionarial.

- Yacimiento de empleo ya ocupado.

En todo caso, el alto porcentaje de economía sumergida puede ser un dato positivo ya que si se normalizara económicamente podría rescatarse como verdadero yacimiento; no obstante, mientras los responsables político-administrativos de la educación no tomen carta en las contrataciones anómalas que realizan las AMPA, la cuestión parece no tener solución, máxime cuando son estas propias autoridades las que aportan unos "carnets" de voluntarios-monitores, a fin de dar una imagen más normalizada de las ilegalidades contractuales cometidas por las asociaciones de padres. Con ello se imposibilita el surgimiento de empresas de servicios y de actividades culturales que podrían coadyuvar a paliar la situación de personas en paro.

A fin de profundizar en tal planteamiento intentaremos a partir de aquí calibrar el número de puestos de trabajo que se podrían ofertar en un sistema educativo con contratación legal y libre del funcionariado destinado a servicios. Para ello extrapolaremos al total del sistema los datos hallados en la muestra analizada y estudiada.

\section{- Área de Servicios. Sistema público. Primaria}

Todas las escuelas de la muestra tenían servicio de portería, por lo que, en el caso de las Baleares, esto supondría 201 puestos de trabajo por este concepto. Asimismo nos encontramos con un promedio de 3 personas de limpieza con cinco horas de trabajo y por centro, lo que supone una oferta de 603 personas, de las cuales, a 8 h por día (jornada completa), sólo podrían trabajar 376. Por lo que respecta a los servicios de cocina y comedor nos hemos encontrado en los centros analizados con 121 cocineros y ayudantes de cocina que trabajan entre 7 y 5 horas al día. La proyección que nos sale desde nuestra muestra para todas las Baleares sería de unos 270 empleados de cocina, lo que a un promedio de 8 horas al día significaría, a nivel de jornada completa, 202,5 empleados, o sea, redondeando 200 puestos de trabajo a tiempo completo. Es decir, el sistema primario público puede ofrecer 777 puestos de trabajo entre porteros, servicio de limpieza y servicos de cocina. 
Los otros servicios son más complejos a la hora de ofertar cálculos. Así, haciendo las extrapolaciones pertinentes en función de nuestra muestra y del número total de escuelas, en cuanto a conductores de autocar se necesitarían 16 en Palma, 12 en Mallorca, 2 en Menorca y 12 en Ibiza, en total 40, lo que ocurre es que al trabajar un máximo de dos horas al día en servicios escolares, significaría, en el mejor de los casos, la creación de 5 puestos de trabajo a dedicación completa, aunque, obviamente, este cálculo es ficticio, ya que es imposible, por motivos de horario, tal dedicación para este tipo de profesionales.

Asimismo, las guarderías en tanto que servicio de las escuelas (que podrían ser unas 15 en el conjunto de islas), los monitores de comedor (que podrían ser más de 275 en todas las Baleares), más los monitores de autobuses que podrían ser 40 , también ofertan trabajos muy atomizados y coincidentes en el horario, lo que imposibilita la generación de empleo estable y con plena dedicación.

Otra cuestión es la del personal encargado del mantenimiento, inexistente en estos niveles a nivel fijo laboral, ya que las reparaciones escolares dependen por lo general de los Ayuntamientos, y en todo caso, los porteros se encargan de las más sencillas y urgentes. De todas formas no podemos pasar por alto, en este campo, la posibilidad de un hipotético yacimiento de empleo que podría tomar forma a través de una cooperativa de servicios.

Como expectativas cara al futuro, y en este capítulo de servicios, los centros - si bien las contestaciones fueron muy pobres - intuyen la necesidad de tener bibliotecas abiertas en la jornada no escolar, así como aumentar el número de guarderías. Por nuestra parte vemos que un día u otro se tendrá que solucionar y ampliar la cuestión administrativa en este tipo de centros, lo que indudablemente daría lugar a un filón importante de empleo, aunque fuesen administrativos compartidos por diversos colegios. Si así sucediere y cada escuela contase con un administrativo al día cada semana, una persona se encargaría de cinco centros, lo que supondría 40 nuevos puestos de trabajo, si bien para el caso de Mallorca y las islas menores habría que contar con el problema de las distancias.

\section{- Área de servicios. Sistema público. Secundaria}

En secundaria pública no encontramos comedores (al menos con personas contratadas fuera de la propia escuela, ya que las tareas de comedor se solapan con las propias de los profesores y alumnos de la especialidad de cocina en los que está implantada esta especialidad); tampoco hay servicios de autocar. En consecuencia sólo podemos referirnos a:

- Conserjes y porteros, que en algunos centros pueden llegar hasta las cuatro personas. Si tenemos en cuenta que en la muestra estudiada nos hemos encontrado con 63 porteros, bien podemos duplicar esta cifra para todos los institutos de Baleares, lo que nos daría unos 120 puestos de trabajo a tiempo completo. 
- Por otra parte, el número de administrativos podría sumar unas 90 personas mínimo (estamos hablando de un total de 54 institutos en todas las Baleares).

- Nos encontramos en nuestra muestra con tres empleadas de biblioteca, que podrían llegar a ser una por instituto (en total 54), pero que en una primera aproximación podrían ser necesarias en la mitad de los mismos, lo que nos daría 27 puestos de trabajo.

- Si tenemos en cuenta que las cantinas o bares están ocupadas por dos o cuatro personas, y aunque sabemos que hay bastantes institutos - sobre todo en Menorca, Ibiza y Formentera - que no disponen de este servicio, podríamos hipotetizar para los 39 institutos restantes, un mínimo de 50 personas a tiempo completo, aunque hay que tener en cuenta que las concesiones de tales establecimientos vienen negociadas a través de la Conselleria encargada de los asuntos educativos.

De todo ello resulta que en total, nuestros cálculos, llegan a los 287 puestos de trabajo en la enseñanza secundaria pública a tiempo completo (120 porteros, 90 administrativos, 27 bibliotecarios y 50 en cantinas), lo que sumados a los encontrados a nivel primario nos hacen un total de 1.064 puestos de trabajo en el sistema público de educación, sólo en el área de servicios y todo ello sin tener en cuenta los conductores de autobuses, los monitores de comedor y autobús, ni el personal de guardería, ni el de mantenimiento. Además debiera tenerse en cuenta que como necesidad más perentoria hemos detectado cara al futuro la creación de comedores escolares. Si incluyésemos en nuestros cálculos todas estas ausencias, estamos convencidos que el sistema público de educación podría dar lugar a un mínimo de 1.100 puestos de trabajo.

\section{- Área de servicios. Sistema concertado-privado}

Calculando de la misma forma, y tras la realidad encontrada, extrapolando nuestras cifras al número total de colegios privados-concertados de las Baleares, tendríamos, para este tipo de centros, los siguientes empleos, teniendo en cuenta que las cifras corresponden respectivamente a Palma, resto de Mallorca, Menorca, Ibiza y Formentera:

\begin{tabular}{|c|c|}
\hline Porteros & $(50+26+10+4)=$ \\
\hline Administración & $(50+40+5+8)=$ \\
\hline Limpieza & $(150+143+22+21)=$ \\
\hline Cocina & $(84+39+0+6)=$ \\
\hline Mantenimiento & $(7+0+0+3)=$ \\
\hline $\begin{array}{l}\text { Enfermería y guardería } \\
\text { TOTAL }\end{array}$ & $(5+0+0+0)=$ \\
\hline
\end{tabular}

Cara al futuro esta cifra podría aumentar ya que en Menorca no existen comedores escolares, así como encargados de mantenimiento que tampoco se 
encuentran en el resto de Mallorca. Por otra parte y como se ve, sólo en Palma se contabilizan guarderías y servicios de enfermería. Además, y en otro orden de cosas, cabe decir que cara al futuro el sistema concertado ve posibilidades de ampliación de los servicios en el desarrollo del horario de las bibliotecas y en la necesidad de contar con mantenimiento de los equipos multimedia.

En resumen, para concluir, y esto ya sería una de las definiciones más fundamentales del nuevo yacimiento hallado, aunque ciertamente ocupado, es que el sistema educativo de las Baleares en el área o aspecto de los servicios puede dar lugar a un mínimo de 1.773 lugares de trabajo a plena dedicación. De todas formas, cara al futuro se debería tener en cuenta las bibliotecas tanto en primaria como en secundaria, los comedores escolares en secundaria, así como la cuestión del mantenimiento y de los administrativos en primaria, asuntos ésos que están aun por resolver, a pesar de que ya se oyen voces a favor, sobre la necesidad de tener apoyo administrativo en las escuelas públicas. Además, no hemos contado ni los conductores de autobús, ni las guarderías fuera de las horas escolares, cuestión ésta que parece ser irá en aumento. Posiblemente con todo ello y de aquí a algunos años se podría aventurar la cifra de 2.000 puestos de trabajo, y un mínimo de más de 17 millones de euros al año de movimiento económico, en una región de poco más de 850.000 habitantes.

- $\quad$ Área de actividades extraescolares

Como se comprenderá, aquí el cálculo es realmente ficticio pues no se pueden extraer jornadas completas de trabajo en lugares laborales que se solapan y que, además, sólo se pueden desarrollar en paralelo a lo largo de unas mismas horas. Sin embargo, intentaremos algunas aproximaciones para poder así captar el interés de este mercado de trabajo de un sorprendente rendimiento económico.

En primer lugar, y a modo de prenotando, decir que finalizado el estudio, y tras analizar todos los tipos de actividades extraescolares o complementarias que se están desarrollando en los centros escolares de las Baleares, presentamos la siguiente sistemática de las mismas pues, apenas sin excepciones, todas ellas nos han cabido en las categorías u órdenes que a continuación presentamos:

- Conocimientos culturales: informática, idiomas, etc.

- Deporte: futbito, fútbol, básquet, judo, voleibol, natación, tenis, etc.

- Psicomotrices: psicomotricidad, manualidades, danza, etc.

- Artísticas: teatro, música, cerámica, dibujo, pintura, decoración, etc.

- Pedagógicas: escuelas de padres, técnicas de estudio... entre otras.

- Lúdicas: salas de juegos, ludoteca, ajedrez, etc.

- Ambientales: talleres de naturaleza, reciclajes. 
Creemos que esta estructura, además de ayudar a clarificar nuestras conclusiones, también podría ser de utilidad a la hora de hipotetizar planes de formación en relación a los nuevos yacimientos de empleo.

En primer lugar, cabe resumir el panorama con el que nos hemos encontrado en referencia a las actividades de formación que ofertan los centros, bien entendido que el primer número se refiere a la suma total de actividades que desarrollan todos los colegios estudiados de cada zona indicada. En cambio, la cifra en paréntesis nos indica el número de actividades diferentes que se organizan en los colegios estudiados de las zonas de referencia; por tanto la comparación entre ambas cifras nos puede dar una idea de la reiteración de actividades por zonas y por tipos de colegios.

\begin{tabular}{lccc} 
& \multicolumn{2}{c}{ Enseñanza pública } & Enseñanza concertada \\
Zona & Primaria & Secundaria & \\
\hline Palma & $119(39)$ & $31(25)$ & $99(36)$ \\
Rest. Mallorca & $288(46)$ & $26(22)$ & $37(20)$ \\
Menorca & $61(25)$ & $10(10)$ & $7(6)$ \\
Ibiza/Formentera & $93(29)$ & $3(3)$ & $16(13)$
\end{tabular}

Para saber cuáles son las actividades que más ofertan los centros, los siguientes cuadros nos informarán de la cuestión, bien entendido que sólo haremos referencia a las diez más solicitadas:

En la enseñanza pública primaria:

\begin{tabular}{llll} 
Palma & Resto Mallorca & Menorca & Ibiza/Formentera \\
\hline Inglés & Inglés & Informática & Informática \\
Teatro & Informática & Inglés & Inglés \\
Básquet & Manualidades & Teatro & Fútbol \\
Manualidades & Básquet & Básquet & Básquet \\
Judo & Voleibol & Manualidacles & Cerámica \\
Música & Teatro & Dibujo & Psicomotric. \\
Informática & Psicomotric. & Fútbol & Manualidades \\
Técn. estudio & Cerámica & Cerámica & Judo \\
Gimn. rítmica & Dibujo & Ajedrez & Tenis \\
Aula juegos & Fútbol & Música & Voleibol \\
& & Psicomotric. & Fútbol-ajedrez
\end{tabular}

O sea, que inglés, informática, manualidades y básquet se repiten en todos los lugares, e incluso se desarrollan en el $40 \%$ de los centros públicos primarios. Además, cabe reseñar que el fútbol, el teatro y la cerámica se reiteran, asimismo, en las tres listas, por lo que consideramos estas siete actividades determinantes en las escuelas públicas de Baleares, que por cierto se corresponden con los grupos de actividades siguientes: 
- Culturales: informática e inglés.

- Deportivas: básquet y fútbol.

- Artísticas: teatro y cerámica.

- Psicomotrices: manualidades.

Obrando de la misma manera en relación a la enseñanza pública de secundaria, nos encontramos las siguientes actividades, si bien, aquí, el número de las indicadas no llega a 10 pues la gran mayoría de ellas sólo son ofrecidas por un único centro. Es decir en todos los IES de Baleares sólo seis actividades se reiteran en diversos centros.

\begin{tabular}{llll} 
Palma & Rest. Mallorca & Menorca & Ibiza/Formentera \\
\hline Informática & Concursos & \\
Teatro & Informática & \\
Cine club & & \\
Inicio Internet & &
\end{tabular}

Como conclusión, decir que la informática se repite en las dos zonas más significativas, aunque también es verdad que es elegida por un centro de Ibiza y que el teatro aparece también en todas las listas. En consecuencia, entre el grupo de materias culturales (informática, inglés, concursos, Internet) y el de materias artísticas (teatro y cine club) es por donde se mueve la oferta más significativa en el sistema público secundario.

En referencia a las escuelas concertadas-privadas, cabe referir lo que sigue:

\begin{tabular}{llll} 
Palma & Resto Mallorca & Menorca & Ibiza \\
\hline Básquet & Inglés & Inglés & Teatro \\
Informática & Básquet & Informática & Voleibol \\
Inglés & Informática & Teatro & Básquet \\
Esc. de pactres & Teatro & Fútbol & \\
Manualidades & Futbito & Música & \\
Teatro & Música & & \\
Cerámica & Ajedrez & & \\
Voleibol & Voleibol & &
\end{tabular}

No introducimos más actividades al no ser discriminativas (sólo realizadas en un solo centro). Como vemos el teatro, el inglés y la informática se encuentran como preferidas en las cuatro zonas (a pesar de que en Ibiza, las dos últimas no se signifiquen o destaquen entre todas las actividades que se desarrollan ya que se dan en un sólo centro). Por lo demás, encontramos el basquet en tres, al igual que el voleibol. En consecuencia, las áreas más afectadas son la cultural (informática e inglés), la deportiva (básquet y voleibol) y la artística (teatro). 
Si cotejamos todos los resultados, vemos que el inglés, la informática, el teatro, y el básquet, son las actividades más generalizadas y por tanto, suponemos, que las más solicitadas en todo el sistema educativo de las Baleares.

En otro orden de cosas, si queremos saber, por áreas, la importancia que éstas ocupan por el numero de actividades que se desarrollan, los resultados son los siguientes:

En PALMA enseñanza pública

$$
\text { (escuela primaria) (escuela secundaria) }
$$

$\begin{array}{lcc}\text { Área Deportiva }= & 32 \text { actividades } & 4 \\ \text { Área Cultural }= & 28 \text { actividades } & 15 \\ \text { Área Artística }= & 23 \text { actividades } & 9 \\ \text { Área Psicomotriz }= & 21 \text { actividades } & 2 \\ \text { Área Pedagógica }= & 5 \text { actividades } & 1 \\ \text { Área Lúdica }= & 5 \text { actividades } & 1 \\ \text { Área Ambiental }= & 2 \text { actividades } & 0 \\ \text { Otras }= & 3 \text { actividades } & \end{array}$

Se nota una incidencia significativa de la actividad cultural en secundaria en detrimento de las deportivas, que pierden, sorprendentemente, casi todo su interés; en cambio, las artísticas manifiestan gran dinamismo ya que conforman un $30 \%$ de todas las actividades de secundaria en Palma.

\section{En RESTO DE MALLORCA}

(escuela primaria) (escuela secundaria)

$\begin{array}{lcc}\text { Área Deportiva }= & 88 & 2 \\ \text { Área Cultural }= & 67 & 9 \\ \text { Área Artística }= & 56 & - \\ \text { Área Psicomotriz }= & 41 & 3 \\ \text { Área Pedagógica }= & 5 & 3 \\ \text { Área Lúdica = } & 27 & 1 \\ \text { Área Ambiental }= & 4 & - \\ \text { Otras } & - & \end{array}$

Si comparamos las dos enseñanzas primarias (Palma y resto de Mallorca) vemos que, de alguna forma, se sigue con la misma tónica, a saber, gran prioridad en el deporte, de los elementos culturales, de las artes y de las actividades lúdicas, si bien, estas últimas, ya a mayor distancia. La diferencia entre enseñanza primaria y secundaria en el resto de Mallorca sigue la misma tónica que en Palma, y si comparamos la secundaria entre Palma y el resto de Mallorca, vemos que el paralelismo es también total, a saber, aumento de las actividades culturales en secundaria así como drástica disminución del deporte, y un número significativo e importante de las actividades artísticas. 


\section{MENORCA}

(escuela primaria) (escuela secundaria)

$\begin{array}{lcc}\text { Área Deportiva }= & 16 & 2 \\ \text { Área Cultural }= & 14 & 4 \\ \text { Área Artística }= & 14 & 2 \\ \text { Área Psicomotriz }= & 9 & 1 \\ \text { Área Pedagógica }= & 2 & 1 \\ \text { Área Lúdica }= & 5 & \\ \text { Área Ambiental }= & 1 & \end{array}$

Como se ve, en Menorca, también se conserva la misma tónica que habíamos encontrado en Palma, y en el resto de Mallorca, tanto en primaria como en secundaria, es decir, el mismo predominio de las actividades en las mismas áreas; en todo caso, indicar que las diferencias entre las cuatro actividades más numerosas son, aquí, apenas existentes, lo que significa un aumento notable y considerable de las actividades artísticas, lo que, culturalmente, se adapta a la tradición de la isla. En referencia a la comparación entre primaria y secundaria, el fenómeno es también idéntico a las otras zonas ya analizadas; es decir, aumento de la actividad cultural, disminución de la deportiva, y alta tónica de las de caracter artístico en secundaria.

IBIZA

$$
\text { (escuela primaria) (escuela secundaria) }
$$

Área Deportiva $=$

Área Cultural $=$

Área Artística $=$

Área Psicomotriz =

Área Pedagógica $=$

Área Lúdica $=$

Área Ambiental =

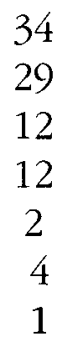

1

1

1

No comparamos la primaria con la secundaria de Ibiza porque, por los escasos parámetros de esta última, consideramos que no tiene ningún sentido. A nivel primario, tampoco en Ibiza, denotamos diferenciación en referencia a los otros territorios, o sea, que en todas las areas geográficas, por lo que se refiere a las actividades extraescolares, en la primaria pública, se concitan de la misma manera, a saber: alto número de actividades deportivas, culturales y artísticas, seguido por las psicomotrices y lúdicas, siendo las demás residuales. 
ANTONIO J. COLOM CAÑELLAS Y CATALINA M." VILANOVA RIPOLL

\section{Enseñanza Concertada privada}

$\begin{array}{lcccc} & \text { Palma } & \text { R. Mca } & \text { Menorca } & \text { Ibiza } \\ \text { Área Deportiva }= & 30 & 18 & 1 & 7 \\ \text { Área Cultural }= & 24 & 9 & 4 & 2 \\ \text { Área Artística }= & 16 & 6 & 2 & 4 \\ \text { Área Psicomotriz }= & 11 & 3 & - & 2 \\ \text { Área Pedagógica }= & 13 & - & - & - \\ \text { Área Lúdica }= & 4 & 2 & - & 1\end{array}$

Se sigue en general con la tónica de las escuelas primarias en todos los territorios, o sea, predominio de las actividades deportivas, culturales y artísticas, siendo las psicomotrices y las lúdicas, secundarias, y las demás, residuales. Podemos pues concluir afirmando que las grandes diferencias se dan entre las actividades de primaria y de la secundaria pública. En todas las demás posibilidades habidas se sigue el mismo modelo para todos los territorios y tipos de escuela. Sólo encontramos una excepción notable, y es que las actividades pedagógicas, en las escuelas concertadas de Palma, se elevan al cuarto lugar, superando a las de carácter psicomotriz y lúdico. El fenómeno creemos tiene su explicación en el mayor contacto que procuran los colegios privados con las familias a fin de cuidar o establecer mayores relaciones con los que en el fondo son los clientes que les mantienen el centro.

Cara a un hipotético programa de formación, o de selección de personal encargado de desarrollar las actividades extraescolares, se requerirían entonces mayores esfuerzos y mayor volumen de recursos en las áreas deportivas, culturales y artísticas; en un segundo plano, encontraríamos la psicomotriz y la lúdica, seguido de forma residual por las áreas pedagógicas (excepto en los centros concertados de Palma) y la de actividades ambientales. En "otros" hemos incluido las actividades de labores, punto y bordados.

Visto el panorama de las enseñanzas complementarias, vamos ahora a abordar la misma cuestión desde una perspectiva más economicista. Tras analizar el número de horas que se dedican a cada una de las actividades de todos los centros estudiados nos sale de promedio dos horas y media por actividad. Sumando todas las actividades que se realizan (en todos los territorios y a todos los niveles de enseñanza, tanto pública como privada-concertada), nos encontramos que son 790 las actividades que se desarrollan en las Baleares, lo que suponen 1.975 horas a la semana de clases complementarias. Ahora bien al encontrar en la enseñanza concertada que se repetían 26 actividades en grupos diferentes habrá que calcular en 2.040 h de clase a la semana, lo que en cuatro semanas - más o menos un mes - nos daría un total de 8.160 horas. Si por otra parte, los monitores reciben entre 15 y 30 euros por hora de clase impartida, y calculando nosotros 22,50 euros por hora (precio medio entre las dos cantidades), nos encontraremos con los siguientes datos:

- Se pagaría en Baleares 45.900 euros por las 2.040 horas semanales de clase. 
- Calculando 4 semanas al mes, el total pagado por tales acciones sería de 183.600 euros, lo que al año (8 meses de curso) supondría 1.468 .800 euros, independientemente de las actividades de verano que cada vez se están organizando más.

- Si calculamos 40 h a la semana como plena ocupación nos daría la cantidad de 51 trabajadores implicados en estas actividades.

- Ello supondría que cada uno de ellos podría llegar a percibir 3.600 euros al mes (a lo que tendríamos que añadir algunas horas más por los días 29 a 31 de cada uno de los meses).

Como se ve son cantidades muy serias para cualquier tipo de economía. Tanto es así que realizando una jornada compartida —sólo 20 h a la semana- 102 personas podrían llegar a cobrar unos 1.800 euros al mes. Es decir, que estamos ante un sector que bien racionalizado y convenientemente compartido y profesionalizado supone un muy potente yacimiento de empleo.

Independientemente de estos cálculos, hay otra cuestión que nos preocupa, y es que, en general, por su importancia económica, y además, por la cantidad de personas que se esconden tras estas actividades, consideramos urgente la normalización y legalización de este mercado laboral. Normalización que vemos difícil pues las AMPA van descubriendo un yacimiento económico importante y de altos beneficios $^{3}$. Cabe pensar que en estos momentos ya hay, en Baleares, cinco colegios públicos en los que todas las actividades, tanto de servicios - con comedor incluido- como las extraescolares, están gestionadas por sendas fundaciones creadas por las AMPA, lo que, no escapa a nadie, es una forma de oficializar formas económicas sumergidas que bien reorientadas conformarían un buen yacimiento de empleo.

A partir de aquí, ¿qué soluciones se pueden arbitrar? Es evidente que un experto en Derecho laboral las conoce perfectamente, por lo que nosotros, legos en el tema, insinuaremos algunas soluciones de sentido común. Por ejemplo, crear cooperativas de servicios escolares (conserjes, limpieza, mantenimiento, administración y actividades complementarias). Es evidente que para cuestiones concretas cabe también el caso de la figura del autónomo. También podrían crearse empresas o sociedades limitadas para ofertar este tipo de servicios, en las que podría darse contratos a tiempo completo o a tiempo parcial (vid. art. 8.2 del texto refundido de la Ley del Estatuto de los Trabajadores). De todas formas, el hecho es que,

3. Un ejemplo lo podemos encontrar en el hecho de que la misma comida que sirve un catering en un colegio público y en otro concertado-privado, cuesta en este último un euro más caro que en el otro. Si en el comedor del colegio concertado-privado se quedan a comer cada día 200 alumnos, las ganancias extra para el AMPA o colegio, son, cada día, del orden de los 200 euros lo que supone 1.000 euros a la semana, o (contando cuatro semanas al mes) un mínimo de 4.000 euros mensuales, que a lo largo de un curso escolar - 8 meses- puede llegar a un mínimo de 32.000 euros año, o sea, bastante más de 5 millones de pesetas de las de antaño. 
con el conocimiento o no de las autoridades administrativas, las AMPA contratan, sin tener licencia para ello, por lo que estaríamos ante un mercado negro de empleo, que por sus beneficios sociales, máxime en una sociedad con alta tasa de paro, se tendría que denunciar.

De una cosa estamos seguros, y es de la cantidad significativa de dinero que mueve el sistema educativo formalizado, independientemente de sus obligaciones escolares (más de 18 millones de euros al año), si bien su provecho social está condicionado por un cambio de mentalidad y que ayuntamientos y administración educativa dejen de funcionariar una serie de actividades que parece absurdo que estén en manos de funcionarios de la Comunidad Autónoma, o de las administraciones locales, como es el caso de porteros, incluso de limpiadoras, o técnicos de mantenimiento; superar estas barreras y que estos empleos saliesen al libre mercado implicaría, indudablemente, un refuerzo para el mercado laboral y la posibilidad de hablar del sistema educativo como un verdadero yacimiento de empleo.

En definitiva, hemos intentado destapar una realidad cotidiana de nuestro sistema educativo que debe tenerse en cuenta en la Teoría Económica de la Educación, y que, a través de las actividades complementarias, nos muestra los puntos críticos de lo que la sociedad de hoy en día supone deben ser los complementos de formación en las edades escolares, lo que nos llevaría a plantear cuestiones muy interesantes en el seno de la sociología de la educación, y en relación a las familias, al papel que en este sentido debe jugar la Teoría de la Educación. No obstante hoy nuestra finalidad se ha centrado más en analizar el papel social que el sistema educativo puede jugar para paliar los déficits del mercado laboral.

\section{BIBLIOGRAFÍA}

Bono, M. J y Jiménez Hernández, E. (1997) Los nuevos yacimientos de empleo, La Factoría, 2.

CaChón, L. (1998a) Creación de empleo y yacimientos de empleo, Gaceta Sindical. Empleo $y$ tiempo de trabajo, 12, 43-49.

- (1998b) Nuevos yacimientos de empleo en España. Madrid, Ministerio de Trabajo y Asuntos Sociales y Fundación Tomillo.

- (1999a) Sobre el desarrollo local y nuevos yacimientos de empleo, Política y Sociedad, $31,117-130$.

- (1999b) Los nuevos yacimientos de empleo, Cuadernos de información económica, 151, 85-93.

COMISIÓN EuROPEA (1995) Iniciativas locales de desarrollo y empleo. Oficina de publicaciones oficiales de las comunidades europeas. Luxemburgo.

Colom, A. J. y ORTE, C. (2001) Gerontología educativa y social. Palma de Mallorca, Universitat de les Illes Balears.

Delors, J. (1993) Libro Blanco. Vid. Comisión de las Comunidades Europeas.

Domíngtez, M. (2000) Nuevos trabajos para un nuevo siglo, Muface, 182, 16-20.

(C) Ediciones Iniversidad de Salamanca

Teor. educ. 14 , 2002, pp. 121-150 
Duque, J. J. (1999) Nuevos Yacimientos de Empleo, Boletín de Estudios Económicos, LIV, 167, 293-299.

Esping-Andersen, G. (1993) Los tres mundos del estado del bienestar. Valencia, Alfons el Magnànim.

Gomis, P. (2000) Consideraciones sobre la estrategia europea de empleo de Amsterdam a Lisboa (junio de 1997 a marzo de 2000), ICE, 784, 103-121.

Jiménez, E.; Barreiro, F. y Sánchez, J. E. (1998) Los nuevos yacimientos de empleo. Barcelona, Fundación CIREM.

Montilla, J. (1996) Desarrollo local y empleo, La Factoria, 1.

Muiños, B. y Cortegiano, G. (1998) Desarrollo a escala local: los nuevos yacimiento de empleo, Revista bibliográfica de Geografia y Ciencias Sociales, 113.

Rifkin, J. (1996) El fin del trabajo. El declive de la fuerza de trabajo global y el nacimiento de la era posmercado. Barcelona, Paidós.

Rubio, R. (2000) Globalización y mercado de trabajo: Retos y oportunidades para la promoción del empleo en el medio local, Scripta nova, 69. 